\title{
Magnetic relaxation and dipole-coupling-induced magnetization in nanostructured thin films during growth: A cluster Monte Carlo study
}

\author{
R. Brinzanik * P.J. Jensen, and K.H. Bennemann \\ Institut für Theoretische Physik, Freie Universität Berlin, Arnimallee 14, D-14195 Berlin, Germany
}

(Dated: November 21, 2018)

\begin{abstract}
For growing inhomogeneous thin films with an island nanostructure similar as observed in experiment, we determine the nonequilibrium and equilibrium remanent magnetization. The single-island magnetic anisotropy, the dipole coupling, and the exchange interaction between magnetic islands are taken into account within a micromagnetic model. A cluster Monte Carlo method is developed which includes coherent magnetization changes of connected islands. This causes a fast relaxation towards equilibrium for irregularly connected systems. We analyse the transition from dipole coupled islands at low coverages to a strongly connected ferromagnetic film at high coverages during film growth. For coverages below the percolation threshold, the dipole interaction induces a collective magnetic order with ordering temperatures of $1-10 \mathrm{~K}$ for the assumed model parameters. Anisotropy causes blocking temperatures of $10-100 \mathrm{~K}$ and thus pronounced nonequilibrium effects. The dipole coupling leads to a somewhat slower magnetic relaxation.
\end{abstract}

PACS numbers: 05.10.Ln, 75.10.Hk, 75.70.Ak, 75.75.+a

\section{INTRODUCTION}

The investigation of low-dimensional magnetic nanostructures has become a very active field of current research. 1 The controlled preparation of different nanostructures allows for the investigation of a variety of interesting magnetic properties ${ }^{2.3 .4}$ The dependence of these properties on the nanostructure and magnetic interactions in such systems is still not well understood. So far, no consistent theoretical analysis has been performed for the magnetic behavior of an ultrathin film during growth, ranging from an island-type structure to a smooth film, in particular the influence of structural disorder on the magnetic properties. In this study, we present Monte Carlo (MC) calculations of the nonequilibrium and equilibrium magnetization of growing inhomogeneous films. Different coverages below and above the percolation threshold and different magnetic interactions are taken into account. This analysis needs a newly developed cluster MC method including coherent rotations of neighboring magnetic islands.

To illustrate the problem, we discuss for instance an ultrathin Co film grown on a $\mathrm{Cu}(001)$ substrate. 5.6 This system shows a perfect layer-by-layer growth for coverages $\Theta>2$ monolayers (ML). Here, the exchange coupling results in a large Curie temperature $T_{\mathrm{C}} \gtrsim$ $350 \mathrm{~K}$. Below the measured percolation coverage of $\Theta_{\mathrm{P}}=$ 1.7 ML, randomly positioned Co islands with a considerable admixture of $\mathrm{Cu}$ atoms are observed, exhibiting a comparably strong remanent magnetization up to temperatures as large as $\sim 150 \mathrm{~K}$. At $\Theta_{\mathrm{P}}$, a jump of $T_{\mathrm{C}}$ of about $100 \mathrm{~K}$ occurs. $\frac{5}{5}$ The important question arises which mechanism causes the strong remanence of an island-type film for coverages $\Theta<\Theta_{P}$. On the one hand, this could be induced by nonequilibrium blocking effects due to single-island anisotropies which impede magnetic relaxation. On the other hand, an equilibrium magnetization can originate from long-range magnetic interactions. We like to discuss these two limiting cases.

An ensemble of magnetically isolated single-domain islands behaves like a superparamagnet. The single-island anisotropy causes a finite time-dependent magnetization below the nonequilibrium blocking temperature $T_{\mathrm{b}}$. For thin $\mathrm{Co} / \mathrm{Cu}(001)$ films this temperature is estimated to be $T_{\mathrm{b}} \approx 5 \mathrm{~K}, 31$ following from the Arrhenius-Néel ansatz $k_{\mathrm{B}} T_{\mathrm{b}}=N K / \ln \left(\tau_{\mathrm{m}} \Gamma_{o}\right) \cdot 7.8$ Note that an island size dispersion will influence the relaxational behavior and $T_{\mathrm{b}}$ sensitively. Second, a finite magnetization may also originate from a collectively ordered state in thermal equilibrium. Such a magnetic state, not necessarily a collinear one, of an ensemble of isolated islands results from longrange magnetic interactions $\frac{9}{-}$ We consider here the magnetic dipole coupling between islands ${ }^{32}$ The corresponding ordering temperature $T_{\mathrm{C}}$ should be comparable to the average dipole energy per island which is, however, difficult to determine for an irregular system. Assuming two neighboring disk-shaped Co islands containing $N$ atoms each, the dipole energy $E_{\text {dip }}$ of this island pair is proportional to $\sqrt{N}$. For $N=1000$, we estimate $E_{\text {dip }} \approx 6 \mathrm{~K}$

Note that both simple estimates for the temperatures $T_{\mathrm{b}}$ and $T_{\mathrm{C}}$ disagree with experimental observations. Hence, improved calculations are needed taking better into account the inhomogeneous film structure characterized by varying island sizes, shapes, and positions. In particular for coverages close to $\Theta_{\mathrm{P}}$, the island coagulation leads to a larger effective island size $N_{\text {eff }}$ and thus to a larger average dipole energy $E_{\text {dip }} \propto \sqrt{N_{\text {eff }}}$. Correspondingly, also the blocking temperature $T_{\mathrm{b}} \propto N_{\text {eff }} K$ due to anisotropy will be larger than the single-island estimate $T_{\mathrm{b}} \approx 5 \mathrm{~K}$. Thus, for a disordered film structure one may expect a remanent magnetization at much higher temperatures than obtained by these simple esti- 
mates.

For the investigation of the magnetic relaxation, the magnetic anistropy and the dipole interaction are taken into account. In case of coagulated islands, the exchange coupling between islands has to be considered as well. For three-dimensional systems of interacting magnetic particles, either longer ${ }^{10}$ as well as shorter ${ }^{11}$ relaxation times as compared to noninteracting ensembles have been calculated. Experimentally, longer relaxation times for increasing interparticle interactions have been measured ${ }^{12,13}$ The existence of a collective, spinglass-like magnetic ordering was discussed $\stackrel{14}{ }$ For twodimensional systems only few investigations have been reported, also indicating longer relaxation times for increasing interaction strengths $\underline{\underline{13.15}}$

In this paper, we report on MC calculations of the nonequilibrium and equilibrium remanent magnetization for nanostructured thin films during growth as function of coverage, temperature, and MC time ${ }^{16.17 .18}$ By application of a modified Ising model, which allows one to take into account magnetization dynamics, the blocking as well as the ordering temperatures are determined. Of particular concern is the consideration of structurally inhomogeneous systems ranging from isolated islands to smooth ferromagnetic films. Numerical simulations are unavoidable since the low symmetry of these systems and the complicated nature of the involved magnetic interactions preclude analytical approaches. However, the application of the common MC technique runs into a severe problem. Assigning a "super" spin to every island magnetic moment (Stoner-Wohlfarth mode ${ }^{19}$ ), conventional single-spin-flip algorithms yield an extremely slow and unrealistic relaxation towards equilibrium for coverages where the islands are partly coagulated ${ }^{34}$ Hence, we apply a cluster-spin-flip algorithm, which includes simultaneous rotations of magnetic moments of connected islands ${ }^{20.21}$ We emphasize that with this method the relaxation behavior and the equilibrium magnetization are calculated efficiently. For all film coverages, the clusterspin-flip method enables an appropriate analysis of the influence of the anisotropy and dipole coupling besides the dominating exchange interaction.

The film growth, the micromagnetic model for the calculation of the magnetic properties, and the cluster MC method are described in Sec. II In Sec. III we test the cluster algorithm for the obtained island nanostructure and present results for the remanent magnetization, as well as for the blocking and ordering temperatures. A conclusion is given in Sec. IV

\section{MODEL AND MC SIMULATION}

\section{A. Growth mode and micromagnetic model}

For the simulation of the island-type growing film, we use the simple solid-on-solid Eden model. ${ }^{17.22}$ Within this model, each additional atom is deposited on an island perimeter site $i$ with probability $p\left(q_{i}, z_{i}\right) \propto$ $\exp \left[A\left(z_{i}\right) \sqrt{q_{i}}\right]$, where $q_{i}$ is the local coordination number and $z_{i}$ the layer index. A bilayer island growth mode is assumed yielding an island structure similar as observed for epitaxial $\mathrm{Co} / \mathrm{Cu}(001) \underline{6}$ A $2 \times 500 \times 500)$ fcc- $(001)$ unit cell with lateral periodic boundary conditions is applied. The island density is $\rho=0.0025$ islands per site, resulting in $Z=625$ randomly distributed islands in the unit cell. For the ratio of binding parameters $\frac{17}{\underline{17}}$ we use $A(1) / A(2)=0.989$.

For the obtained atomic structures, a micromagnetic model for the total (free) energy of a system of interacting magnetic islands is applied: ${ }^{16.17}$

$$
\begin{aligned}
E & =-\frac{1}{2} \sum_{i>j} L_{i j}(\Theta) \gamma_{i j}(\Theta, T) \boldsymbol{S}_{i} \boldsymbol{S}_{j} \\
& +\sum_{i>j} \frac{\mu_{i}(\Theta, T) \mu_{j}(\Theta, T)}{r_{i j}^{5}}\left[r_{i j}^{2} \boldsymbol{S}_{i} \boldsymbol{S}_{j}-3\left(\boldsymbol{r}_{i j} \boldsymbol{S}_{i}\right)\left(\boldsymbol{r}_{i j} \boldsymbol{S}_{j}\right)\right] \\
& -\sum_{i} N_{i}(\Theta) K_{i}(\Theta, T)\left(S_{i}^{x}\right)^{2},
\end{aligned}
$$

with $\Theta$ being the film coverage and $T$ the temperature. Each magnetic island with $N_{i}(\Theta)$ atoms is treated as a Stoner-Wohlfarth particle $\frac{19}{}$ with a single giant magnetic moment $\mu_{i}=\mu_{\text {at }} m_{i} N_{i}$, whose direction is confined to the film plane, where $\mu_{\text {at }}$ is the atomic magnetic moment. The unit vector $\boldsymbol{S}_{i}=\boldsymbol{\mu}_{i} / \mu_{i}$ characterizes the magnetic moment direction of the $i$ th island. The first term in Eq. (1) represents the magnetic domain wall energy between connected islands, with $L_{i j}$ the number of bonds between islands $i$ and $j$, and $\gamma_{i j}$ the domain wall energy per atomic bond. The second term is the long-range magnetic dipole interaction between the island magnetic moments $\mu_{i}$ where $r_{i j}=\left|\boldsymbol{r}_{i}-\boldsymbol{r}_{j}\right|$ is the distance between the centers of islands $i$ and $j$. The point-dipole energy is calculated by applying the Ewald summation technique over all periodically arranged unit cells of the thin film 18.23 The last term denotes the uniaxial in-plane anisotropy energy with $K_{i}$ the anisotropy per atomic spin. Due to this anisotropy we allow for only two stable directions for each island moment $\left(S_{i}^{x}= \pm 1\right)$. Thus, our system refers to a modified Ising model, for which during magnetization reversal a possible anisotropy energy barrier is taken into account, hence allowing the consideration of magnetization dynamics. Finally, due to the finite exchange coupling $J$ between neighboring atomic spins, the internal island magnetization $m_{i}(\Theta, T)$ is taken into account within a mean-field approximation. This leads to temperaturedependent effective anisotropy coefficients $K_{i}(\Theta, T)$ and domain wall energy densities $\gamma_{i j}(\Theta, T)$, as described in greater detail in Ref. 17.

Equation (11) describes a system of dipole-coupled single islands at low film coverages $\Theta \ll \Theta_{P}$ as well as a connected ferromagnetic film at high coverages $\Theta \gg \Theta_{P}$. We point out that the transition between these extremal cases during the film growth is described within the same model. The assumption of individual magnetic islands 
with varying interactions is a good approximation as long as the system is laterally nanostructured, whereas for smooth films (here $\Theta \approx 2.0 \mathrm{ML}$ ) it represents an unphysical discretization of the system.

\section{B. Cluster MC method}

The magnetic equilibrium and nonequilibrium properties are calculated by performing kinetic cluster MC simulations. Especially, close to the percolation coverage $\Theta_{\mathrm{P}}$, most of the magnetic islands are connected to neighboring islands and form large but still finite clusters. A single-spin-flip (SSF) algorithm ${ }^{22}$ for such an irregular atomic structure yields a very slow relaxation towards thermodynamic equilibrium, since subsequent flips of island magnetic moments in this cluster of connected islands, as considered by SSF updates, are strongly hindered by the exchange energy, see Ref. 34. Thus, a rotation of the entire island cluster is very unlikely, and its dependence on dipole interaction and anisotropy is strongly underestimated. For an improved simulation of the magnetic relaxation, a coherent or simultaneous rotation of the spins in these clusters has to be taken into account. For this purpose, we propose a cluster-spin-flip (CSF) algorithm ${ }^{20.21}$ in the present study.

In a first step of each MC update, a cluster $\mathcal{C}_{\nu}$ consisting of $\nu$ connected islands is constructed by the following scheme:

(a) Choose randomly a single island $i$, representing the first (smallest) island cluster $\mathcal{C}_{1}=\{i\}$.

(b) Add a random second island $j$ which is connected to island $i\left(L_{i j} \neq 0\right)$, forming the second island cluster $\mathcal{C}_{2}=\{i, j\}$.

(c) Construct subsequently larger island clusters $\mathcal{C}_{\nu}$ by adding a randomly chosen island to the preceding cluster $\mathcal{C}_{\nu-1}$, provided that this island is connected to at least one of the $\nu-1$ islands of $\mathcal{C}_{\nu-1}$.

(d) Continue this construction procedure till either no additional adjacent islands are present or if a maximum allowed number $\lambda_{\max }$ of islands in the cluster is reached. From this procedure, we obtain a set of $\lambda \leq \lambda_{\max }$ island clusters $\left\{\mathcal{C}_{1}, \ldots, \mathcal{C}_{\lambda}\right\}$.

(e) Out of this set choose one cluster $\mathcal{C}_{\nu}$ with weight $\omega_{\nu}$ for probing $\left(\sum_{\nu} \omega_{\nu}=1\right)$.

A Monte Carlo step (MCS) is defined by the usual condition that $Z$ islands in the system are probed. Employing a cluster $\mathcal{C}_{\nu}$ containing $\nu$ islands considers the portion $\nu / Z$ of the system in a single update. To ensure that probing large clusters does not dominate the relaxation process, we assign the weight $\omega_{\nu}=1 / \nu$ for choosing $\mathcal{C}_{\nu}$ out of the set $\left\{\mathcal{C}_{1}, \ldots, \mathcal{C}_{\lambda}\right\}$. This definition implies that within a single MCS no additional relaxation channels are opened by the consideration of island cluster flips ${ }^{35}$ We emphasize that not only is the largest possible island cluster $\mathcal{C}_{\lambda}$ probed for flipping, but all island clusters out of the corresponding set are considered. The island moments within an island cluster need not to be parallel.

In the second step of each update, all $\nu$ island spins of the chosen cluster $\mathcal{C}_{\nu}$ are probed for a coherent flip. The corresponding flip rate $\Gamma_{\nu}$ is calculated in the usual way as if these $\nu$ connected islands form a single large island, 17 From Eq. (1), the magnetic energy of this island cluster as function of the in-plane angle $\phi$ is given by

$$
\epsilon_{\nu}(\phi)=\frac{E_{\nu}(\phi)}{\mathcal{K}_{\nu}}=-2 h_{\nu} \cos \phi-\cos ^{2} \phi,
$$

with the reduced magnetic field

$$
h_{\nu}=\frac{\sum_{k l} S_{k}^{x} S_{l}^{x}\left[L_{k l} \gamma_{k l}+\mu_{k} \mu_{l}\left(4 x_{k l}^{2}-2 y_{k l}^{2}\right) / r_{k l}^{5}\right]}{4 \mathcal{K}_{\nu}}
$$

and the total anisotropy energy of the cluster $\mathcal{K}_{\nu}=$ $\sum_{k=1}^{\nu} N_{k} K_{k}$. Here, the $k$ sum runs over all spins inside, and the $l$ sum over all spins outside the island cluster $\mathcal{C}_{\nu}$. We have neglected the dipole sums $\sum_{k l}\left(x_{k l} y_{k l}\right) / r_{k l}^{5}$ which are usually smaller than the sums $\sum_{k l}\left(x_{k l}\right)^{2} / r_{k l}^{5}$ and $\sum_{k l}\left(y_{k l}\right)^{2} / r_{k l}^{5}$.

The Ising-like states $S_{i}^{x}= \pm 1$ of $\mathcal{C}_{\nu}$ represent either energy minima which are separated by an anisotropy energy barrier, or refer to an energy maximum and minimum. The respective energy barriers for the forward and backward transitions are given by

$$
\begin{array}{rll}
\Delta E_{\nu}^{(1)}=\left(h_{\nu} \pm 1\right)^{2} \mathcal{K}_{\nu} & \text { for } & \left|h_{\nu}\right|<1 \\
\Delta E_{\nu}^{(2)}= \pm 4 h_{\nu} \mathcal{K}_{\nu} & \text { for } & \left|h_{\nu}\right| \geq 1
\end{array}
$$

The flip rate $\Gamma_{\nu}^{(1)}$ of the island spin cluster $\mathcal{C}_{\nu}$ to overcome $\Delta E_{\nu}^{(1)}$ is calculated from the common Arrhenius-Néel ansatz ${ }^{7.8}$ We use a constant prefactor $\Gamma_{o}=10^{9} \mathrm{sec}^{-1}$ which determines the time unit of the magnetic relaxation in kinetic MC simulations ${ }^{36}$ The latter case is treated with the usual Metropolis-type rate, using the same prefactor ${ }^{22}$

The growing thin film is characterized by a large amount of nonequivalent lattice sites, corresponding to a large number of different interaction parameters. Since little is known about these values, we use in our simulation averaged quantities for the magnetic parameters which are fixed as follows, using as an example the $\mathrm{Co} / \mathrm{Cu}(001)$ thin-film system. The atomic magnetic moments are set to $\mu_{\mathrm{at}}=2.0 \mu_{\mathrm{B}} \stackrel{24}{ }$ The domain wall energy $\gamma$ is adjusted to give the observed Curie temperature of the ferromagnetic long-range order of $T_{\mathrm{C}}=355 \mathrm{~K}$ of a 2-ML $\mathrm{Co} / \mathrm{Cu}(001)$ film,,$\frac{5}{\underline{3}}$ yielding $\gamma=5.6 \mathrm{meV} /$ bond. The exchange interaction for the calculation of the internal island magnetic ordering is set equal to $J=$ $7.0 \mathrm{meV} /$ bond $\stackrel{17}{ }$ For the uniaxial anisotropy, two different values $K=0.1$ and $0.01 \mathrm{meV} /$ atom are investigated.

In this study, we determine the remanent magnetization $M_{\mathrm{rem}}(\Theta, T, t)=\sum_{i} N_{i} S_{i}^{x}(t) m_{i}(\Theta, T) / \sum_{i} N_{i}$ of the growing thin film, where $t$ is the $\mathrm{MC}$ time in units of MCS. The simulation starts from a completely aligned island spin state. The choice of this initial state refers to experiments which saturate the magnetic system by an 
external magnetic field and determine the remanent magnetization after removal of the field ${ }^{3.5}$ We have no evidence that magnetic arrangements end up in metastable states during relaxation when starting from a saturated state. In addition, we calculate the equilibrium magnetization $M_{\text {eq }}(\Theta, T)$, which is obtained by averaging $M_{\text {rem }}(\Theta, T, t)$ over a range of 500 MCS after the system has become equilibrated. $M_{\text {rem }}(\Theta, T, t)$ and $M_{\text {eq }}(\Theta, T)$ are averaged over at least 20 different structural runs. The magnetizations are given in units of a saturated monolayer (i.e., $\Theta=1 \mathrm{ML}$ ) at $T=0$. Since the finitesized unit cell undergoes eventually total magnetic reversals during $\mathrm{MC}$ probing, accidental cancellation of a finite $M_{\text {rem }}$ and $M_{\text {eq }}$ during structural and temporal averaging may occur. To avoid this, we use in this study merely the absolute values $\left|M_{\mathrm{rem}}\right|$ and $\left|M_{\mathrm{eq}}\right|$.

\section{RESULTS AND DISCUSSION}

\section{A. Island growth and CSF algorithm}

Snapshots of the atomic structure during thin film growth, resulting from our growth model, are shown in Ref. 17. The resulting static atomic structure is similar to the one observed for the $\mathrm{Co} / \mathrm{Cu}(001)$ system. 6 In the initial stages of growth, randomly located islands with almost rectangular shapes are obtained. With increasing film coverages, the single islands start to coagulate and form island clusters with a still finite size. By analysing the percolation probability using the HoshenKopelman algorithm, we yield a percolation coverage of about $\Theta_{\mathrm{P}} \approx 0.9 \mathrm{ML}{ }^{25.26}$ Continued film growth leads to a connected thin film. In this coverage range, the system still exhibits a distinct irregular nanostructure. Isolated island clusters vanish rapidly upon further adatom deposition. The coverage $\Theta=2.0 \mathrm{ML}$ corresponds to a smooth magnetic film with two closed layers.

At first, we investigate the effect of the cluster-spinflip MC method on the simulation of the remanent magnetization $\left|M_{\text {rem }}(\Theta, T, t)\right|$. Here we consider only the exchange interaction. For a strongly connected film $\left(\Theta_{\mathrm{P}} \ll \Theta=1.8 \mathrm{ML}\right.$ we test whether by use of CSF $\left|M_{\text {rem }}(\Theta, T, t)\right|$ relaxes into the correct equilibrium value $\left|M_{\mathrm{eq}}(\Theta, T)\right|$, starting from a fully aligned state. As can be seen from Fig. 1(a), this condition is fulfilled, since different maximum allowed numbers $\lambda_{\max }$ of islands in the cluster lead to the same equilibrium value as for the single-spin-flip MC method. The larger the number $\lambda_{\max }$ is, the slower is the relaxation. This property is caused by the fact that in this coverage and temperature range the magnetic relaxation is mainly provided by flips of single islands or small island clusters. As mentioned in Sec. IIB no additional relaxation channels are opened by use of CSF, hence the number of single-spin-flip attempts becomes reduced in favor of unprobable cluster-spin-flip ones. Examples for the error bars are also given. The statistical error, which is similar for all forthcoming figures, results mainly from averaging over different structural realizations of the unit cell and could be reduced by using larger unit cells.

We point out that the main improvement of the CSF with respect to the SSF method is obtained for coverages $\Theta \lesssim \Theta_{\mathrm{P}}$, characterized by a considerable amount of island cluster formation, and which is very difficult to be studied analytically. In Fig. 1(b), the magnetic relaxation for $\Theta=0.8 \mathrm{ML}$ is depicted using different $\lambda_{\max }$. The equilibrium magnetization $M_{\text {eq }}^{0}(\Theta, T)$ should vanish for $\Theta<\Theta_{P}$, since long-range magnetic interactions are neglected here. Due to the use of the absolute value a finite but small $\left|M_{\text {eq }}^{0}(\Theta, T)\right|$ is obtained in our calculations. The SSF algorithm exhibits an extremely slow magnetic relaxation toward $\left|M_{\mathrm{eq}}^{0}\right|$. Even after $10^{6} \mathrm{MCS}$ the remanent magnetization is relaxed only to $\left|M_{\text {rem }}^{0}\right|=0.60$. The reason is that this method considers very unfavorable intermediate states. Already the allowance of a few coherently flipping island spins results in a much faster relaxation. The relaxational behavior converges rapidly with increasing $\lambda_{\max }$. Using the CSF with $\lambda_{\max }=50$ or larger the equilibrium is reached already after $\sim 100 \mathrm{MCS}$. To obtain a fast equilibration, the closer the coverage to $\Theta_{\mathrm{P}}$ the larger the value chosen for $\lambda_{\max }$. The CSF algorithm leads to a much faster equilibration also for coverages $\Theta \gtrsim \Theta_{P}$. In this coverage range, island clusters are still present which have only weak links to other clusters. In the following investigations, we set $\lambda_{\max }$ equal to the number of single islands $Z=625$, except for coverages $\Theta \gg \Theta_{P}$ where we yield a better performance for $\lambda_{\max }=100$.

Hence, by use of the SSF algorithm the exchange interaction grossly dominates the MC simulations for strongly inhomogeneous systems. This is avoided by applying CSF, allowing thus for the investigation of the effect of the much weaker anisotropy and dipole interaction.

\section{B. Effect of interactions}

First, we study the combined effect of the dipole and the exchange interaction on the film magnetization for coverages $\Theta<\Theta_{P}$. We determine equilibrium properties which within our model are not influenced by the anisotropy. In Fig. 2(a), we present $\left|M_{\text {rem }}(\Theta, T, t)\right|$ for different temperatures $T$ as a function of MC time $t$. The coverage is assumed to be $\Theta=0.8 \mathrm{ML}$. Starting from the fully aligned state $\left|M_{\text {rem }}(\Theta, T, t=0)\right|=0.8$, the remanent film magnetization relaxes fast to its equilibrium value. For the assumed temperatures the dipole coupling leads to a net magnetization $\left|M_{\text {rem }}\right|>\left|M_{\text {rem }}^{0}\right|$ where for $\left|M_{\text {rem }}^{0}\right|$ the dipole interaction is neglected. After several hundred MCS equilibration is obtained for the dipole-coupling induced $\left|M_{\text {rem }}\right|$ which then stays stable within the simulation time. We emphasize that it is impossible to obtain these and the following results with conventional SSF algorithms.

In Fig. 2(b), the equilibrium magnetization 
$\left|M_{\text {eq }}(\Theta, T)\right|$ is shown as function of temperature $T$ for different coverages $\Theta$. For low temperatures, clearly a magnetic ordering due to the dipole interaction is seen. The larger the coverage is, the larger is the ordering effect, since with an increasing $\Theta$ the average island cluster size and thus the average dipole coupling energy increases. Above the ordering temperatures $T_{\mathrm{C}}(\Theta)$, the magnetizations $\left|M_{\mathrm{eq}}(\Theta, T)\right|$ reach the corresponding values $\left|M_{\mathrm{eq}}^{0}(\Theta, T)\right|$ as calculated without the dipole interaction. Due to the use of absolute values, $\left|M_{\text {eq }}^{0}(\Theta, T)\right|$ stays always finite. $T_{\mathrm{C}}(\Theta)$ is estimated by extrapolating the linear part of $\left|M_{\mathrm{eq}}\right|$ to $\left|M_{\mathrm{eq}}^{0}\right|$. The ordering temperature even for the largest investigated coverage is quite small, yielding $T_{\mathrm{C}} \approx 6 \mathrm{~K}$ for $\Theta=0.8 \mathrm{ML}$. The rounding of $\left|M_{\text {eq }}\right|$ near the ordering temperature is caused by (i) the finite unit cell size, (ii) the use of the absolute value, (iii) the presence of island size and -position dispersions, and (iv) the average over 20 different realizations of the unit cell.

The existence of a long-range magnetic ordering due to the dipole interaction has been calculated for periodic lattices $\stackrel{27}{2}$ In our study, we find that also within an irregular island system below the percolation threshold the dipole interaction leads to a magnetic ordering, indicated by a net magnetization $\left|M_{\mathrm{eq}}\right|>\left|M_{\mathrm{eq}}^{0}\right|$. Such a collective state is expected to be spin-glass-like, as discussed for three-dimensional magnetic particle systems,$\stackrel{14}{,}$ and which needs a further investigation. We remark that the obtained ordering temperatures will increase by considering a noncollinear island magnetization beyond $S_{i}^{x}= \pm 1,28$ by taking into account the finite island extension for the dipole interaction beyond the point-dipole approximation,,$\frac{29}{.}$ or by consideration of densely packed three-dimensional particles.

In Fig. 3. we investigate the influence of the magnetic anisotropy and the exchange interaction on the magnetic relaxation for coverages $\Theta<\Theta_{\mathrm{P}}$. Here, the dipole coupling is neglected. In Fig. 3(a), the remanent magnetization $\left|M_{\mathrm{rem}}(\Theta, T, t)\right|$ is given as function of MC time $t$ for different temperatures $T$ and for $K=0.01 \mathrm{meV} /$ atom. The coverage is assumed to be $\Theta=0.8$ ML. Starting from the fully aligned state, at first $\left|M_{\text {rem }}(\Theta, T, t)\right|$ drops rapidly due to relaxation of single islands and small island clusters. The further relaxation happens much more slowly since here larger island clusters have to be reversed. For $T \gtrsim 25 \mathrm{~K}$, the magnetization $\left|M_{\mathrm{rem}}(\Theta, T, t)\right|$ reaches within the depicted time range the curve $\left|M_{\text {rem }}^{0}\right|$ as calculated for $K=0$.

In Figs. 3(b) and 3(c), we show $\left|M_{\text {rem }}(\Theta, T, t)\right|$ after $t=1000$ MCS for different coverages as function of temperature, using the anisotropy parameters $K=$ 0.01 and $0.1 \mathrm{meV} /$ atom. With increasing temperature, the magnetization $\left|M_{\text {rem }}(\Theta, T, t)\right|$ approaches the equilibrium value $\left|M_{\mathrm{eq}}^{0}\right|$. The corresponding blocking temperatures $T_{\mathrm{b}}(\Theta, K)$ are obtained by extrapolating the linear part of $\left|M_{\text {rem }}\right|$ to $\left|M_{\text {eq }}^{0}\right|$. A rounding of $\left|M_{\text {rem }}\right|$ near $T_{\mathrm{b}}$ is observed due to the same reasons as discussed in connection with Fig. 2 .
We emphasize that for a connected island structure an increase of the anisotropy $K$ by a factor of 10 does not necessarily lead to an increase of $T_{\mathrm{b}}(\Theta, K)$ by the same factor as obtained from the Stoner-Wohlfarth model 19 This is caused by internal cluster excitations, i.e., creation or motion of domain walls inside island clusters. To discuss this, we have performed additional calculations for an infinite domain wall energy $\gamma$, indicated by the full lines in Figs. B(b) and 3(c), hence allowing only for coherent island cluster rotations. Above a certain temperature, the curves for finite and infinite $\gamma$ deviate, since then internal cluster excitations become effective. For $K=0.01 \mathrm{meV} /$ atom and coverage $\Theta=0.6 \mathrm{ML}$, the difference between these curves is small, thus the magnetic relaxation happens mainly via coherent rotation. In contrast, for $\Theta=0.8 \mathrm{ML}$ or for the larger anisotropy $K=0.1 \mathrm{meV} /$ atom, obviously both relaxation processes are present.

Which relaxation process is effective at a given temperature is determined by its energy barrier $\Delta E$. For a coherent rotation of an isolated island cluster, $\Delta E$ is given by its total anisotropy energy $\mathcal{K}_{\nu}$. In contrast, $\Delta E$ for an internal cluster excitation consists of both the anisotropy of the actually reversed islands and the domain wall energy, see Eqs.(44) and (5). By closer investigation, we found that a particular relaxation process becomes effective above a temperature amounting to $5-10 \%$ of $\Delta E$. In the temperature ranges $T<40 \mathrm{~K}$ for $K=$ $0.01 \mathrm{meV} /$ atom and $T<100 \mathrm{~K}$ for $K=0.1 \mathrm{meV} /$ atom, each internal cluster excitation consists mainly of reversing only one or two islands. For markedly larger temperatures, the internal cluster excitations will become more complex, depending in a complicated way on the nanostructure.

The influence of the dipole coupling on the relax-

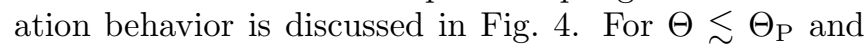
$K=0.01 \mathrm{meV} /$ atom, the dipole interaction results in a small increase of $\left|M_{\text {rem }}\right|$ and $T_{\mathrm{b}}$. Thus, the dipole interaction leads to a slower magnetic relaxation. Interestingly, this effect is visible in the whole temperature range up to $T_{\mathrm{b}}$, and is not limited to those small temperatures where the dipole coupling induces a magnetic ordering, see Fig. 2 The increase of $\left|M_{\text {rem }}\right|$ will become larger if a stronger dipole coupling is assumed, for example for larger island magnetic moments. Experimentally, a similar effect was observed for two-dimensional arrays of interacting magnetic nanoparticles with random anisotropy axes $\frac{13}{} \mathrm{~A}$ more detailed investigation of this property is needed.

Next, we investigate the magnetization for coverages above the percolation threshold, $\Theta>\Theta_{\text {P. }}$. In Fig. [5 the equilibrium magnetization $\left|M_{\mathrm{eq}}(\Theta, T)\right|$ is shown as function of temperature for different coverages. Here, the exchange coupling causes a fast magnetic relaxation and a strong ferromagnetic long-range order. For large coverages and at low temperatures, the behavior of $\left|M_{\mathrm{eq}}\right|$ is reigned by the decrease of the internal island magnetization $m_{i}(\Theta, T)$, whereas at elevated temperatures a 
strong decay of $\left|M_{\mathrm{eq}}\right|$ is caused by the disturbance of the island spin alignment. The resulting ordering temperatures $T_{\mathrm{C}}(\Theta)$ are deduced from the inflection points of $\left|M_{\mathrm{eq}}(\Theta, T)\right|$. In addition, for $\Theta=1.0 \mathrm{ML}$ and 1.2 ML we show the nonequilibrium remanent magnetization $\left|M_{\text {rem }}\right|$ after $t=1000 \mathrm{MCS}$, considering $K=$ $0.01 \mathrm{eV} /$ atom. The corresponding blocking temperatures $T_{\mathrm{b}}(\Theta)$ are markedly larger than $T_{\mathrm{C}}(\Theta)$ in the coverage range $\Theta \gtrsim \Theta_{P}$, where the nanostructure of the percolated thin film is still very irregular and nonequilibrium effects due to anisotropy barriers are pronounced. For films with larger coverages, having a higher connectivity between islands, the exchange coupling results in a fast magnetic relaxation and thus the temperature difference between $T_{\mathrm{b}}$ and $T_{\mathrm{C}}$ is small. A very weak ordering effect due to the dipole interaction is visible only for very low temperatures and coverages $\Theta \gtrsim \Theta_{\mathrm{P}}$. Here, still a few isolated islands or island clusters exist which are coupled to the percolating cluster by the dipole interaction.

In Fig. 6] we summarize the most important results of this study. The (nonequilibrium) blocking temperature $T_{\mathrm{b}}(\Theta, K)$ and the (equilibrium) ordering temperature $T_{\mathrm{C}}(\Theta)$ are presented as functions of coverage $\Theta$ in the whole investigated growth range. For a better visualization, a logarithmic temperature scale is applied. $T_{\mathrm{b}}$ is determined for two different anisotropies $K=0.01$ and $0.1 \mathrm{meV} /$ atom and $t=1000 \mathrm{MCS}$. Below the percolation coverage $\Theta_{\mathrm{P}}$, the dipole interaction induces small ordering temperatures $T_{\mathrm{C}}$ of the order of $1-10 \mathrm{~K}$ for the assumed model parameters. Due to the coagulation of islands with increasing coverage the exchange interaction becomes more important, since it couples single islands to magnetically aligned large clusters. This results in a strong increase of $T_{\mathrm{C}}$ in particular close to $\Theta_{\mathrm{P}}$. This behavior has been observed in experiments on $\mathrm{Co} / \mathrm{Cu}(001)$ ultrathin films (" $T_{\mathrm{C}}$-jump") $\stackrel{5.6}{5}$ For percolated thin films, the ordering temperature is of the order of $100-300 \mathrm{~K}$ and is, within the accuracy of our calculations, exclusively determined by the exchange coupling. The slope of $T_{\mathrm{C}}(\Theta)$ for $\Theta>\Theta_{\mathrm{P}}$ is not as steep as for $\Theta<\Theta_{\mathrm{P}}$. In addition, we show the ordering temperature $T_{\mathrm{C}}$ due to dipole interaction by neglecting the exchange coupling between islands. Evidently, a distinct variation of $T_{\mathrm{C}}$ near $\Theta_{P}$ is not obtained in this case.

The nonequilibrium behavior as caused by the anisotropy $K$ differs strongly for coverages below and above $\Theta_{\mathrm{P}}$. Due to the slow relaxation of the irregular atomic structure for $\Theta<\Theta_{\mathrm{P}}$, a blocking temperature $T_{\mathrm{b}}(\Theta)$ is obtained which is an order of magnitude larger than $T_{\mathrm{C}}(\Theta)$ resulting from the dipole interaction. Evidently, $T_{\mathrm{b}}$ depends on the anisotropy $K$ and the MC time $t$. On the other hand, for $\Theta>\Theta_{\mathrm{P}}$, the relaxation is accelerated by the exchange interaction. With increasing $\Theta$ the remanent magnetization reaches the equilibrium value within $t=1000$ MCS, hence $T_{\mathrm{b}}(\Theta)$ merges into $T_{\mathrm{C}}(\Theta)$.

Recently, a mean field theory (MFT) for the dipolecoupling induced magnetic ordering temperature has been performed, using a simplified growth model $\underline{6}$ A qualitatively similar behavior of $T_{\mathrm{C}}(\Theta)$ as compared to the present $\mathrm{MC}$ calculations was obtained, in particular the strong variation of $T_{\mathrm{C}}$ near $\Theta_{\mathrm{P}}$ due to the exchange interaction between coagulated islands. Evidently, MFT yields much larger values for $T_{\mathrm{C}}(\Theta)$ for such low-dimensional systems especially for $\Theta<\Theta_{P}$, since thermal fluctuations are neglected.

In the following, we discuss our results in connection with measurements on $\mathrm{Co} / \mathrm{Cu}(001)$ ultrathin films. Although several model parameters are chosen in accordance with this system, a full quantitative comparison cannot be drawn yet. The main reason is that the observed intermixing of $\mathrm{Co}$ adatoms with $\mathrm{Cu}$ substrate atoms is not taken into account within our growth model due to the incomplete knowledge of the resulting atomic morphology. The measured percolation threshold $\Theta_{\mathrm{P}} \approx 1.7 \mathrm{ML}$ is much larger than the one as obtained with the growth parameters used by us.

To investigate solely the effect of an enlarged $\Theta_{\mathrm{P}}$, we have performed additional simulations simply by taking into account magnetic islands with up to three atomic layers, yielding the observed $\Theta_{\mathrm{P}}$. Then for a coverage $\Theta=1.6 \mathrm{ML}$, the dipole coupling induces a ordering temperature $T_{\mathrm{C}} \approx 50 \mathrm{~K}$. The corresponding blocking temperature for $K=0.01 \mathrm{meV}$ and $t=1000$ MCS is obtained to be $T_{\mathrm{b}} \approx 150 \mathrm{~K}$. Hence, we find that for the assumed growth modes and magnetic parameters the blocking temperatures are always markedly larger than the ordering temperatures. These temperatures are comparable with measured temperatures $T_{\mathrm{C}} \approx 150 \mathrm{~K}$ for coverages slightly below $\Theta_{\mathrm{P}}{ }^{\frac{5}{5}}$ In addition, to draw a quantitative comparison with the $\mathrm{Co} / \mathrm{Cu}(001)$ system a fourfold symmetry of the in-plane anisotropy has to be taken into account. We expect that the above stated general behavior of the magnetic relaxation and ordering obtained with a uniaxial anisotropy will not be changed.

\section{CONCLUSION}

In this study, we have calculated the nonequilibrium and equilibrium remanent magnetization of growing ultrathin films, using a cluster Monte Carlo method. An island-type nanostructure with a nonuniform distribution of island sizes, shapes, and locations was investigated. Within a micromagnetic model (modified Ising model) the single-island magnetic anisotropy, the dipole coupling, and the exchange interaction between magnetic islands were taken into account. We have analysed the transition from dipole-coupled islands for film coverages below the percolation threshold $\Theta_{\mathrm{P}}$ towards a connected ferromagnetic film above $\Theta_{P}$ with increasing film coverage.

For coverages $\Theta<\Theta_{\mathrm{P}}$, the dipole interaction leads to an equilibrium net magnetization refering to a collectively ordered state. A small ordering temperature $T_{\mathrm{C}}$ of about $1-10 \mathrm{~K}$ results for the assumed model parameters. 
$T_{\mathrm{C}}$ increases strongly near $\Theta_{\mathrm{P}}$ due to exchange interaction which aligns coagulated islands. On the other hand, the anisotropy induces a pronounced nonequilibrium remanent magnetization which may be visible in experiment even after long waiting times. The corresponding blocking temperature $T_{\mathrm{b}}$ is obtained to be of the order of $10-100 \mathrm{~K}$, which is always markedly larger than $T_{\mathrm{C}}$. Approaching $\Theta_{\mathrm{P}}$, the proportionality between blocking temperature and anisotropy is no longer valid due to relaxation via internal island cluster excitations. A nonequilibrium remanent magnetization due to anisotropy is visible also for coverages $\Theta \gtrsim \Theta_{\mathrm{P}}$ where the film is still very irregular. For smoother films at larger coverages the exchange interaction induces a fast magnetic relaxation towards equilibrium.

We have obtained these results with a cluster-spin-flip algorithm which takes into account coherent magnetic rotations of island clusters. This method leads to a very fast and more realistic magnetic relaxation towards equilibrium in the coverage range with an irregular nanostructure. Our results cannot be achieved by conventional single-spin-flip algorithms. The suggested CSF algorithm can be applied also to other inhomogeneous spin systems such as diluted magnets and spin glasses.
Several possible improvements of our micromagnetic model are pointed out. In this study, we have used Isinglike states $S_{i}^{x}= \pm 1$. By applying continuously varying spins $\boldsymbol{S}_{i}$, noncollinear magnetic arrangements can be analysed, ${ }^{28}$ allowing one to also determine the effects of an external magnetic field for these strongly inhomogeneous films. In particular, the movement of magnetic domain walls can be investigated. Furthermore, various magnetic nanostructures like chains and stripes 3 can be studied easily by a proper variation of the parameters of the Eden-type growth model. Anisotropies with, e.g., a four-fold in-plane symmetry will be considered. Finally, the relaxation laws and times of the remanent magnetization can be investigated for such thin film systems. ${ }^{3}$

\section{Acknowledgments}

This work was supported by the Deutsche Forschungsgemeinschaft, SFB 290, TP A1, and 450, TP C4. Discussions with Prof. K. Baberschke and Dr. J. Lindner are acknowledged.
* electronic address: brinzani@physik.fu-berlin.de

1 F. J. Himpsel, J. E. Ortega, G. J. Mankey, and R. F. Willis, Adv. Phys. 47, 511 (1998).

2 S. Sun, C. B. Murray, D. Weller, L. Folks, and A. Moser, Science 287, 198 (2000).

3 J. Shen, R. Skomski, M. Klaua, H. Jenniches, S. S. Manoharan, and J. Kirschner, Phys. Rev. B 56, 2340 (1997).

4 O. Pietzsch, A. Kubetzka, M. Bode, and R. Wiesendanger, Phys. Rev. Lett. 84, 5212 (2000).

5 U. Bovensiepen, P. Poulopoulos, W. Platow, M. Farle, and K. Baberschke, J. Magn. Magn. Mater. 192, L386 (1999).

${ }^{6}$ P. Poulopoulos, P. J. Jensen, A. Ney, J. Lindner, and K. Baberschke, Phys. Rev. B 65, 064431 (2002), and references therein.

7 L. Néel, Ann. Géophys. (C.N.R.S.) 5, 99 (1949).

8 W. F. Brown, Jr., J. Appl. Phys. 30, 130S (1959).

${ }^{9}$ R. P. Cowburn, A. O. Adeyeye, and M. E. Welland, New J. Phys. 1, 16.1 (1999).

10 J. L. Dormann, D. Fiorani, and E. Tronc, J. Magn. Magn. Mater. 202, 251 (1999), and references therein.

11 M. F. Hansen and S. Mørup, J. Magn. Magn. Mater. 184, 262 (1998), and references therein.

12 H. Mamiya, I. Nakatani, and T. Furubayashi, Phys. Rev. Lett. 80, 177 (1998).

13 P. Poddar, T. Telem-Shafir, T. Fried, and G. Markovich, Phys. Rev. B 66, 060403(R) (2002).

14 T. Jonsson, P. Svedlindh, and M. F. Hansen, Phys. Rev. Lett. 81, 3976 (1998).

15 D. K. Lottis and R. M. White, Phys. Rev. Lett. 67, 362 (1991).

16 M. E. Hilo, K. O. O'Grady, and R. W. Chantrell, J. Appl. Phys. 76, 6811 (1994).
17 R. Brinzanik, P. J. Jensen, and K. H. Bennemann, J. Magn. Magn. Mater. 238, 258 (2002).

18 R. Brinzanik, Ph.D. thesis, Freie Universität Berlin (2003), electronic version at URL http://www.diss.fuberlin.de/2003/253/indexe.html.

19 E. C. Stoner and E. P. Wohlfarth, Nature (London) 160, 650 (1947).

${ }^{20}$ U. Wolff, Phys. Rev. Lett. 62, 361 (1989).

21 S. W. Davis, W. McCausland, H. C. McGahagan, and M. Widom, Phys. Rev. E 59, 2424 (1999).

22 D. P. Landau and K. Binder, A Guide to Monte Carlo Simulations in Statistical Physics (Cambridge University Press, Cambridge, 2000).

23 P. J. Jensen, Ann. Phys. (Leipzig) 6, 317 (1997).

24 P. Srivastava, F. Wilhelm, A. Ney, M. Farle, H. Wende, N. Haack, G. Ceballos, and K. Baberschke, Phys. Rev. B 58, 5701 (1998).

25 D. Stauffer, Introduction to Percolation Theory (Taylor \& Francis, London and Philadelphia, 1985).

26 J. Hoshen and R. Kopelman, Phys. Rev. B 14, 3438 (1976).

27 K. De'Bell, A. B. MacIsaac, and J. P. Whitehead, Rev. Mod. Phys. 72, 225 (2000).

28 P. J. Jensen and G. M. Pastor, Phys. Stat. Sol. (a) 189, 527 (2002).

29 P. J. Jensen and G. M. Pastor, New J. Phys. 5, 68 (2003).

30 B. Heinrich, J. F. Cochran, M. Kowalewski, J. Kirschner, Z. Celinski, A. S. Arrot, and K. Myrtle, Phys. Rev. B 44, 9348 (1991).

31 We have assumed the measured in-plane anisotropy $K=$ $0.01 \mathrm{meV} /$ atom ${ }^{30}$ a typical number of atoms $N=1000$ per Co island at $\Theta<\Theta_{\mathrm{P}}, \underline{6}$ a measurement time $\tau_{\mathrm{m}}=100 \mathrm{sec}$, and the attempt frequency $\Gamma_{o}=10^{9} \mathrm{sec}^{-1}, \frac{7.8}{\mathrm{~B}} k_{\mathrm{B}}$ is the Boltzmann constant. 
32 In addition to the magnetic dipole coupling other longrange interactions can be present such as the indirect exchange (RKKY-) interaction in case of metallic substrates, and the superexchange for insulating substrates.

33 Consider two disk-shaped Co islands with $N$ atoms each. Assume that the distance $R$ between their centers is comparable to their diameters, $R \approx D \propto a_{o} \sqrt{N}$. Then the point-dipole energy of this island pair is $E_{\text {dip }} \approx$ $\left(N \mu_{\mathrm{at}}\right)^{2} / R^{3} \approx 0.623\left(\mu_{\mathrm{at}}^{2} / a_{o}^{3}\right) \sqrt{N} \approx 6 \mathrm{~K}$, with $\mu_{\mathrm{at}}=$ $2.0 \mu_{\mathrm{B}}$ the atomic magnetic moment measured for a $2-\mathrm{ML}$ Co film, ${ }^{24} \mu_{\mathrm{B}}$ the Bohr magneton, $a_{o}=2.5 \AA$ the Co interatomic distance, and $N=1000$.

${ }^{34}$ Consider for instance the magnetic rotation $\uparrow \uparrow \rightarrow \downarrow \downarrow$ of an isolated island cluster consisting of two connected islands. Assuming Ising-like magnetic moments, inevitably the single-spin-flip method applies a subsequent rotation of the single island spins through the intermediate state $\uparrow \downarrow$. However, such a process is very unlikely due to the large increase of exchange energy of this intermediate state.
Obviously, a proper treatment of magnetic relaxation requires the inclusion of a coherent rotation of the island pair. This is performed within a cluster-spin-flip algorithm which takes into account such simultaneous spin rotations of connected islands.

35 The described CSF algorithm satisfies the condition of detailed balance. This is guaranteed by the fact that the probabilities for construction and choice of the cluster $\mathcal{C}_{\nu}$ are the same for both flip directions, and by the used flip rates which already obey detailed balance. Ergodicity is maintained since any spin state can be reached due to the allowance of single-spin flips.

36 A constant attempt frequency $\Gamma_{o}$ is widely applied in literature. The justification of this approach is given in Refs. 7 and 8 where $\Gamma_{o}$ is calculated using different approaches. $\Gamma_{o}$ is found to vary weakly with temperature and local fields. Note that the applied transition rates depend only weakly on the exact value of $\Gamma_{o}$. 

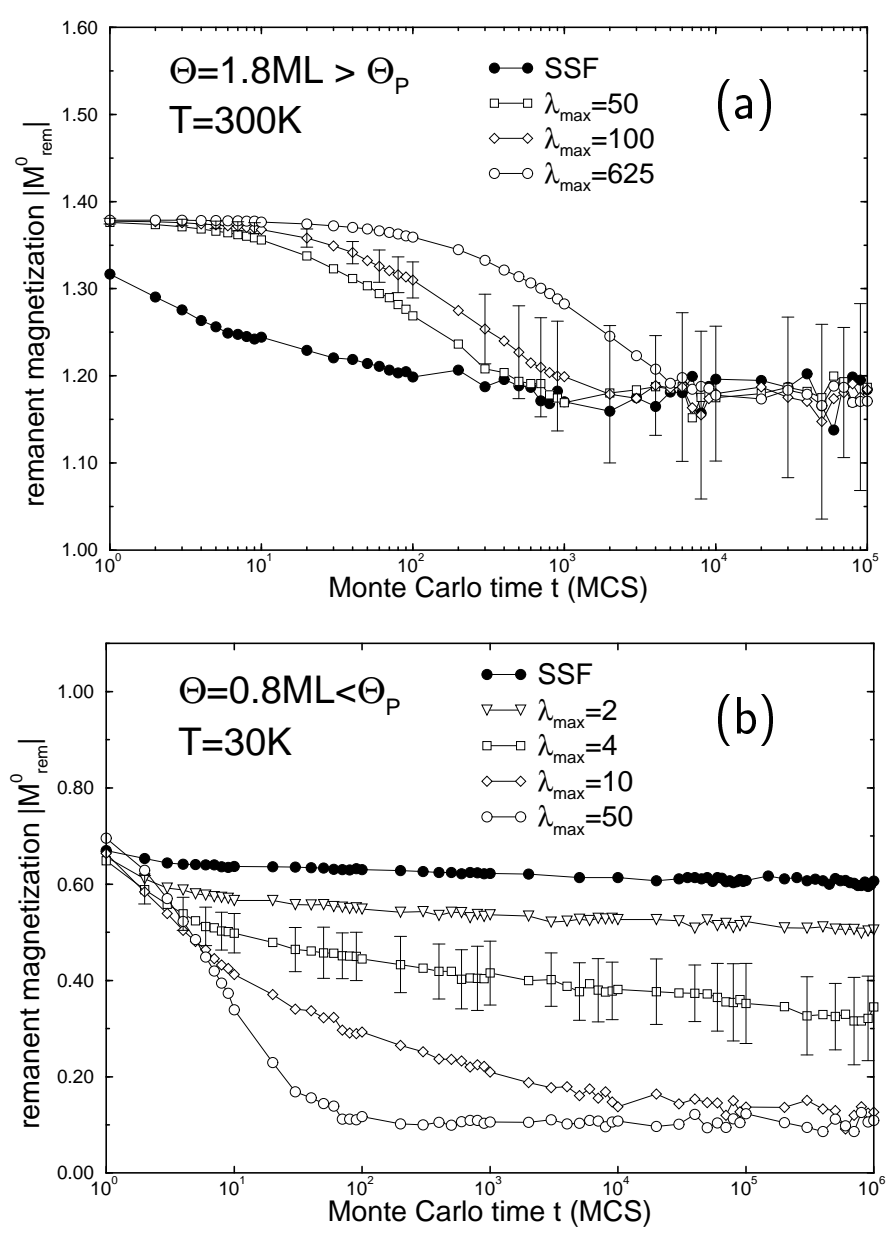

FIG. 1: Comparison of the calculated film magnetization using the single-spin-flip (SSF) and cluster-spin-flip (CSF) algorithm. Only the exchange coupling between magnetic islands is considered. The remanent magnetization $\left|M_{\mathrm{rem}}^{0}\right|$ as function of MC time $t$ for two film coverages (a) $\Theta=1.8 \mathrm{ML}$ and (b) $\Theta=0.8 \mathrm{ML}$ above and below the percolation coverage $\Theta_{\mathrm{P}} \approx 0.9 \mathrm{ML}$ is shown. Within the CSF method, different maximum numbers $\lambda_{\max }$ of coherently flipping island magnetic moments are used. 

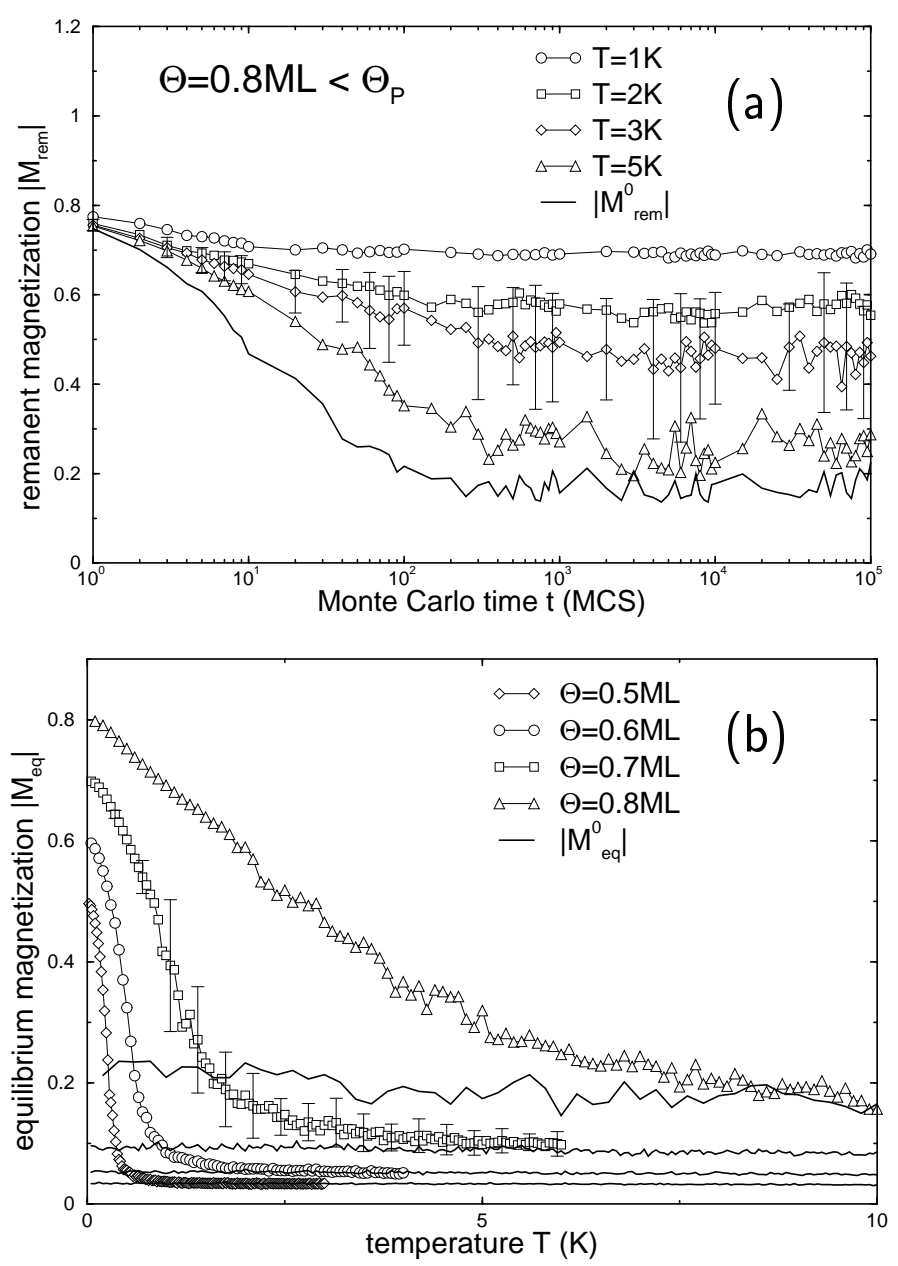

FIG. 2: Long-range magnetic ordering due to dipole interaction for coverages $\Theta$ below the percolation coverage $\Theta_{P}$. Only dipole and exchange interactions are included. The quantities $\left|M_{\mathrm{rem}}^{0}\right|$ and $\left|M_{\mathrm{eq}}^{0}\right|$ neglect the dipole interaction. (a) Remanent magnetization $\left|M_{\mathrm{rem}}\right|$ as function of MC time $t$ for $\Theta=0.8 \mathrm{ML}<\Theta_{\mathrm{P}}$ and different temperatures $T$. (b) Equilibrium magnetization $\left|M_{\text {eq }}\right|$ as function of temperature $T$ for different coverages $\Theta<\Theta_{\mathrm{P}}$. 

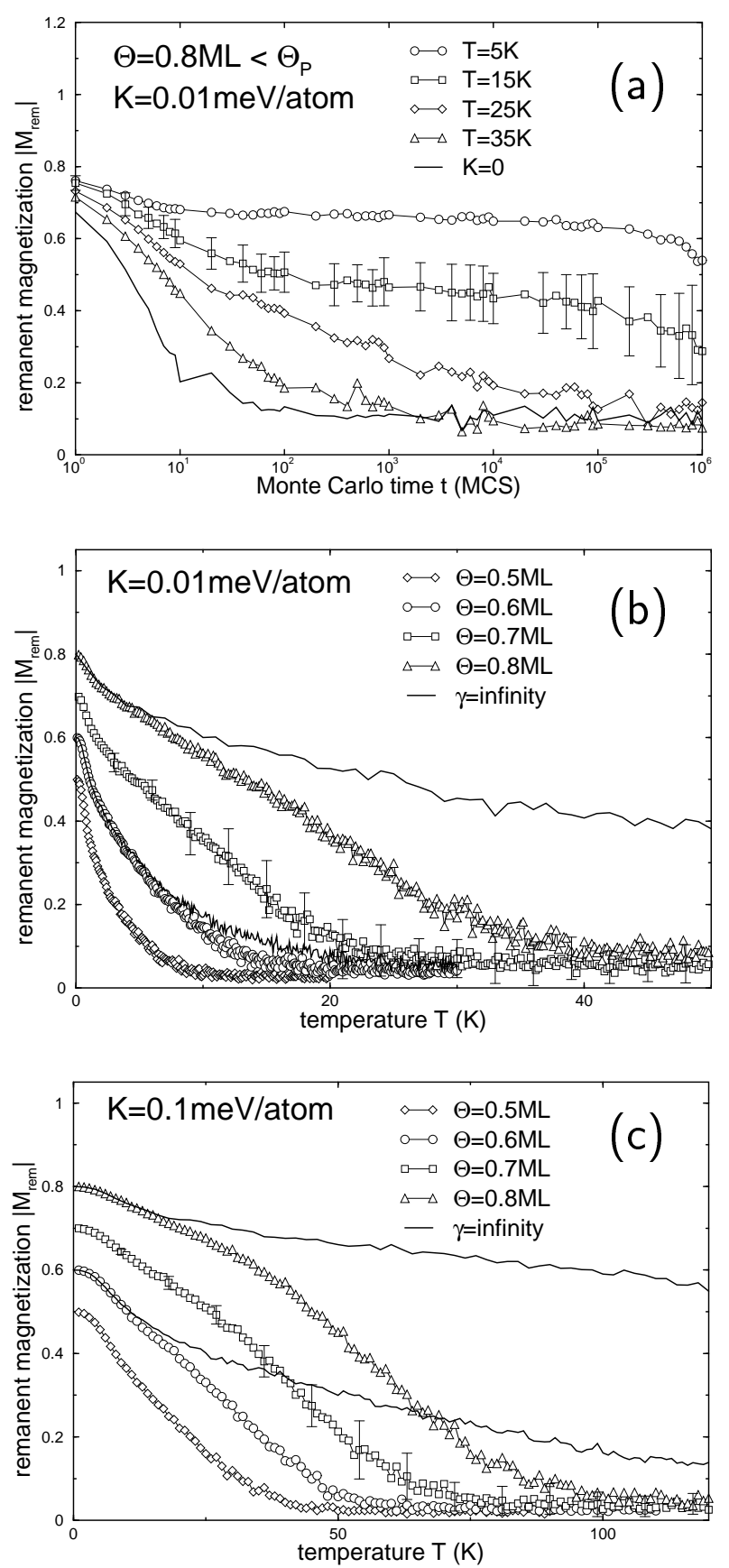

FIG. 3: Relaxation of the remanent magnetization for coverages $\Theta$ below the percolation coverage $\Theta_{\mathrm{P}}$. Only anisotropy and exchange interaction are taken into account. (a) Remanent magnetization $\left|M_{\mathrm{rem}}\right|$ as function of $\mathrm{MC}$ time $t$ for $\Theta=0.8 \mathrm{ML}<\Theta \mathrm{P}$, anisotropy $K=0.01 \mathrm{meV} /$ atom, and different temperatures $T$. The full line refers to $K=0$ and $T=35 \mathrm{~K}$. Furthermore, (b) and (c) show the remanent magnetization $\left|M_{\mathrm{rem}}\right|$ after $t=1000 \mathrm{MCS}$ as function of temperature $T$ for $K=0.01$ and $0.1 \mathrm{meV} /$ atom. The full lines are calculated with an infinite domain wall energy $\gamma$. 


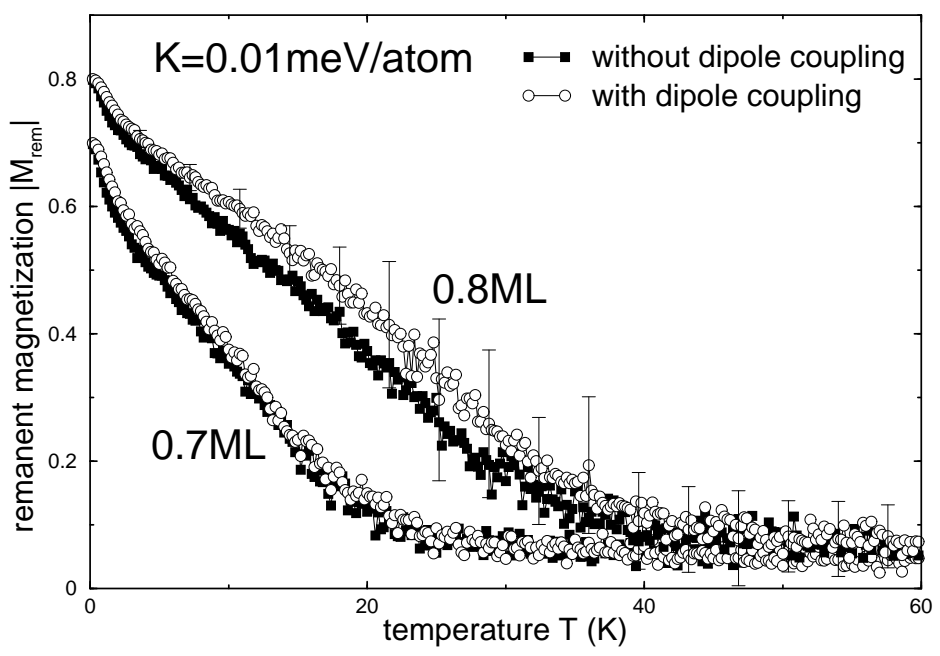

FIG. 4: Influence of magnetic anisotropy and dipole coupling on the remanent magnetization $\left|M_{\text {rem }}(T)\right|$ as shown in Fig. 3(b). Results for two coverages $\Theta$ below the percolation coverage $\Theta_{P}$ are presented.

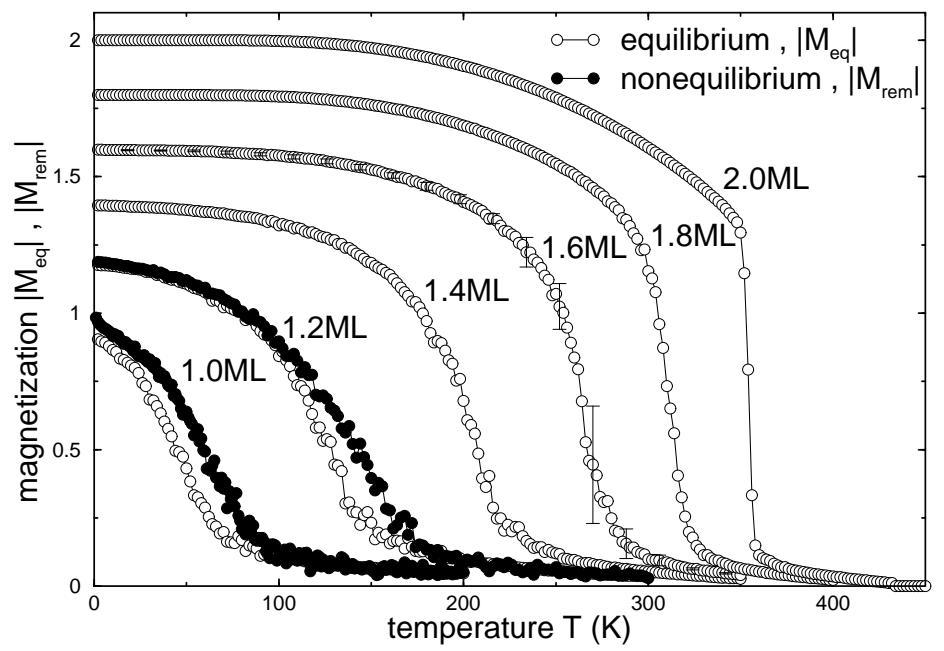

FIG. 5: Long-range ferromagnetic ordering due to exchange interaction for different coverages $\Theta$ above the percolation coverage $\Theta_{\mathrm{P}}$. The equilibrium magnetization $\left|M_{\mathrm{eq}}\right|$ is shown as function of temperature $T$. For comparison, for $\Theta=1.0$ and $1.2 \mathrm{ML}$ also the remanent magnetization $\left|M_{\text {rem }}\right|$ after $t=1000 \mathrm{MCS}$ is depicted for an anisotropy $K=0.01 \mathrm{meV} / \mathrm{atom}$. 


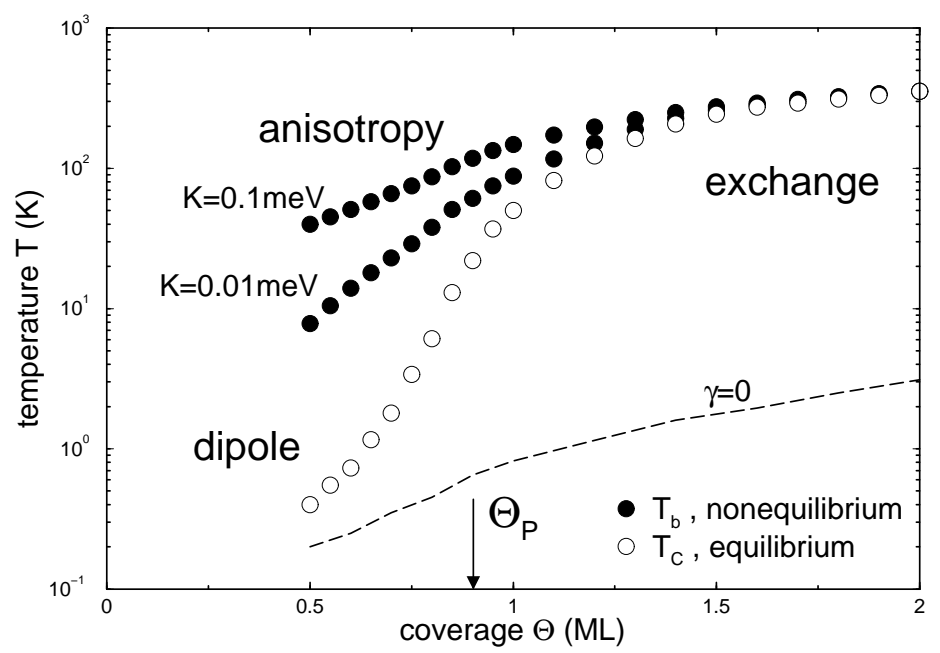

FIG. 6: Semi-logarithmic plot of the magnetic ordering temperature $T_{\mathrm{C}}$ and the blocking temperature $T_{\mathrm{b}}$ as functions of the film coverage $\Theta$. The temperatures $T_{\mathrm{C}}$ and $T_{\mathrm{b}}$ are extracted from the preceding figures. The entire investigated coverage range below and above the percolation coverage $\Theta_{\mathrm{P}}$ is shown. The different magnetic interactions dominate in different coverage and temperature ranges as indicated. The dashed curve refers to the dipole-coupling-induced ordering temperature neglecting the domain wall energy $\gamma$ between islands. 